\title{
Correction: Jaramillo, F.; et al. Priorities and Interactions of Sustainable Development Goals (SDGs) with Focus on Wetlands. Water 2019, 11, 619
}

\author{
Fernando Jaramillo ${ }^{1,2,3, * \mathbb{C}}$, Amanda Desormeaux ${ }^{4}$, Johanna Hedlund ${ }^{2}$, James W. Jawitz ${ }^{4}(\mathbb{D}$, \\ Nicola Clerici ${ }^{5}{ }^{(0)}$, Luigi Piemontese ${ }^{2}{ }^{-}$, Jenny Alexandra Rodríguez-Rodriguez ${ }^{6}$, \\ Jesús Adolfo Anaya ${ }^{7}{ }^{\circledR}$, Juan F. Blanco-Libreros ${ }^{8}{ }^{\oplus}$, Sonia Borja ${ }^{1}$, Jorge Celi ${ }^{9}$, \\ Sergey Chalov ${ }^{10,11}{ }^{\circledR}$, Kwok Pan Chun ${ }^{12}$, Matilda Cresso ${ }^{1}$, Georgia Destouni ${ }^{1}{ }^{1}$, \\ Shimelis Behailu Dessu ${ }^{13}$, Giuliano Di Baldassarre ${ }^{14}{ }^{(\mathbb{C}}$, Andrea Downing ${ }^{2}$, Luisa Espinosa ${ }^{6}{ }^{(\mathbb{C}}$, \\ Navid Ghajarnia ${ }^{1}{ }^{(0}$, Pierre Girard ${ }^{15}$, Álvaro G. Gutiérrez ${ }^{16}{ }^{\circledR}$, Amy Hansen ${ }^{17}$, Tengfei Hu ${ }^{18}$, \\ Jerker Jarsjö ${ }^{1}$, Zahra Kalantari ${ }^{1}{ }^{1}$, Adnane Labbaci ${ }^{19}{ }^{\circledR}$, Lucia Licero-Villanueva ${ }^{6}$, \\ John Livsey ${ }^{1}{ }^{\circledR}$, Ewa Machotka ${ }^{20}$, Kathryn McCurley ${ }^{4}{ }^{\circledR}$, Sebastián Palomino-Ángel ${ }^{7}{ }^{\circledR}$, \\ Jan Pietron ${ }^{1}$, René Price ${ }^{13}{ }^{\circledR}$, Sorain J. Ramchunder ${ }^{21} \oplus$, Constanza Ricaurte-Villota ${ }^{6} \oplus$, \\ Luisa Fernanda Ricaurte ${ }^{22}$, Lula Dahir ${ }^{23}$, Erasmo Rodríguez ${ }^{24}$, Jorge Salgado ${ }^{25}$,
} A. Britta K. Sannel ${ }^{1}{ }^{\circledR}$, Ana Carolina Santos ${ }^{26}$, Samaneh Seifollahi-Aghmiuni ${ }^{1}{ }^{\complement}$, Ylva Sjöberg ${ }^{1}$, Lian Sun ${ }^{1,27}$, Josefin Thorslund ${ }^{1}{ }^{1}$, Guillaume Vigouroux ${ }^{1}$, Lan Wang-Erlandsson ${ }^{2}$, Diandian Xu ${ }^{1,28}$ (1), David Zamora ${ }^{24}$, Alan D. Ziegler ${ }^{21}$ and Imenne Åhlén ${ }^{1}$

1 Department of Physical Geography and Bolin Centre for Climate Research, Stockholm University, SE-106 91, 10691 Stockholm, Sweden; sonia.borja@natgeo.su.se (S.B.); matildacresso@gmail.com (M.C.); gia.destouni@natgeo.su.se (G.D.); navid.ghajarnia@natgeo.su.se (N.G.); jerker.jarsjo@natgeo.su.se (J.J.); zahra.kalantari@natgeo.su.se (Z.K.); john.livsey@natgeo.su.se (J.L.); jan.pietron@natgeo.su.se (J.P.); britta.sannel@natgeo.su.se (A.B.K.S.); samaneh.seifollahi@natgeo.su.se (S.S.-A.); ylva.sjoberg@natgeo.su.se (Y.S.); sunlian@mail.bnu.edu.cn (L.S.); josefin.thorslund@natgeo.su.se (J.T.E.); guillaume.vigouroux@natgeo.su.se (G.V.); diandianws@outlook.com (D.X.); imenne.ahlen@natgeo.su.se (I.Å.)

2 Stockholm Resilience Centre, Stockholm University, 10691 Stockholm, Sweden; johanna.hedlund@su.se (J.H.); luigi.piemontese@su.se (L.P.); andrea.downing@su.se (A.D.); lan.wang@su.se (L.W.-E.)

3 Baltic Sea Centre, Stockholm University, SE-106 91, 10691 Stockholm, Sweden

4 Soil and Water Sciences Department, University of Florida, 2191, McCarty Hall, Gainesville, FL 32611, USA; amandamdesormeaux@gmail.com (A.D.); jawitz@ufl.edu (J.W.J.); klmccurley@ufl.edu (K.M.)

5 Biology Program, Faculty of Natural Sciences and Mathematics, Universidad del Rosario, Kr 26 No 63B-48, Bogotá D.C. 111221, Colombia; nicola.clerici@urosario.edu.co

6 Instituto de Investigaciones Marinas y Costeras de Colombia “José Benito Vives de Andreis"—INVEMAR, Santa Marta 470006, Colombia; alexandra.rodriguez@invemar.org.co (J.A.R.-R.);

luisa.espinosa@invemar.org.co (L.E.); lucia.licero@invemar.org.co (L.L.-V.); constanza.ricaurte@invemar.org.co (C.R.-V.)

7 Facultad de Ingeniería, Universidad de Medellín, Carrera $87 \mathrm{~N}^{\circ}$ 30-65, Medellín 050026, Colombia; janaya@udem.edu.co (J.A.A.); spalomino@udem.edu.co (S.P.-Á.)

8 Instituto de Biología, Universidad de Antioquia, Medellín 050010, Colombia; juan.blanco@udea.edu.co

9 Grupo de Investigación de Recursos Hídricos y Acuáticos, Universidad Regional Amazónica IKIAM, 150150 Tena Kilómetro 7, vía Muyuna, Atacapi, Ecuador; jorge.celi@ikiam.edu.ec

10 Faculty of Geography, Lomonosov Moscow State University, 119571 Moscow, Russia; srchalov@geogr.msu.ru or chalov@ukw.edu.pl

11 Institute of Geography, Kazimierz Wielki University, 85-064 Bydgoszcz, Poland

12 Department of Geography, Hong Kong Baptist University, Hong Kong; kpchun@hkbu.edu.hk

13 Southeast Environmental Research Center, Florida International University, Miami, FL 33199, USA; ssetegn@fiu.edu (S.B.D.); pricer@fiu.edu (R.M.P.)

14 Centre of Natural Hazards and Disaster Science, CNDS, Department of Earth Sciences, Uppsala University, 75236 Uppsala, Sweden; giuliano.dibaldassarre@geo.uu.se 
15 Departamento de Botânica e Ecologia, Universidade Federal de Mato Grosso (UFMT) and Centro de Pesquisa do Pantanal, Cuiabá-MT 78060-900, Brazil; pierregirard1301@gmail.com

16 Departamento de Ciencias Ambientales y Recursos Naturales Renovables, Facultad de Ciencias Agronómicas, Universidad de Chile, Santiago 8820808, Chile; bosqueciencia@gmail.com

17 Civil, Environmental and Architectural Engineering Department, University of Kansas, Lawrence, KS 66045, USA; amy.hansen@ku.edu

18 Hydrology and Water Resources Department, Nanjing Hydraulic Research Institute, Nanjing 210029, China; soaringhu@outlook.com

19 Faculty of Sciences, Ibn Zohr University, Agadir 80000, Morocco; labbaciadnane@gmail.com

20 Department of Asian, Middle Eastern and Turkish Studies, Stockholm University, SE-106 91 Stockholm, Sweden; ewa.machotka@su.se

21 Geography Department, National University of Singapore, Singapore 119077, Singapore; geosjr@nus.edu.sg (S.J.R.); geoadz@nus.edu.sg (A.D.Z.)

22 Independent Consultant, 8000 Zurich, Switzerland; ricaurte.luisa@gmail.com

23 Independent Consultant, 11122 Stockholm, Sweden; luladahir@gmail.com

24 Civil and Agricultural Department, Universidad Nacional de Colombia, Bogotá 11001, Colombia; earodriguezs@unal.edu.co (E.R.); dazamoraa@unal.edu.co (D.Z.)

25 Grupo de Palinología y Paleoecología Tropical, Departamento de Ciencias Biológicas, Universidad de Los Andes, Bogotá 111711, Colombia; Universidad Católica de Colombia, Bogotá 111311, Colombia; j.salgado@uniandes.edu.co

26 Instituto de Investigación de Recursos Biológicos Alexander von Humboldt, Bogotá 111311, Colombia; asantos@humboldt.org.co

27 State Key Laboratory of Water Environment Simulation, School of Environment, Beijing Normal University, Beijing 100875, China

28 College of Water Conservancy and Hydropower Engineering, Hohai University, Nanjing 210098, China

* Correspondence: fernando.jaramillo@natgeo.su.se

Received: 18 October 2019; Accepted: 12 December 2019; Published: 25 December 2019

The authors wish to make the following correction to this paper [1]: The author name "Zahra Kalantary" should be "Zahra Kalantari".

We apologize for any inconvenience caused to the readers.

\section{Reference}

1. Jaramillo, F.; Desormeaux, A.; Hedlund, J.; Jawitz, J.W.; Clerici, N.; Piemontese, L.; Rodríguez-Rodriguez, J.A.; Anaya, J.A.; Blanco-Libreros, J.F.; Borja, S.; et al. Priorities and interactions of Sustainable Development Goals (SDGs) with focus on wetlands. Water, 2019, 11, 619. 